\title{
Two Cases of Chronic Idiopathic Intestinal Pseudo-obstruction with Different Clinical Features
}

\author{
Byoung Hwan Lee, M.D. ${ }^{1}$, Nayoung Kim, M.D. ${ }^{1 *}$, Sung-Bum Kang, M.D. ${ }^{2}$, Kyoung-Ho Lee, M.D. ${ }^{3}$, Jane C. Oh, M.D. ${ }^{4}$ \\ Sun-Mi Kim, M.D. ${ }^{1}$, Young Soo Park, M.D. ${ }^{1}$, and Dong Ho Lee, M.D. ${ }^{1}$ \\ Departments of ${ }^{1}$ Internal Medicine, ${ }^{2}$ Surgery, and ${ }^{3}$ Radiology, Seoul National University Bundang Hospital, Seongnam, Gyeonggi-do, Korea, \\ ${ }^{4}$ Younsei Plus Clinic, Seongnam, Gyeonggi-do, Korea
}

Chronic intestinal pseudo-obstruction (CIPO) is a rare disorder characterized by a severe impairment of gastrointestinal propulsion in the absence of mechanical obstruction. We experienced a case of chronic pseudo-obstruction in the initial phase mimicking acute pseudo-obstruction, which was treated medically. This ongoing case was compared to another recurrent and intractable case successfully treated with surgery and diagnosed as hypoganglionosis. These two cases showed different clinical features and therapeutic approaches for CIPO; one with the first episode of CIPO mimicking Ogilvie's syndrome; the other with recurrent episodes of CIPO with typical features. In conclusion, CIPO is a difficult disorder with various clinical manifestations and different treatment modalities, therefore individualized diagnostic and therapeutic approaches are needed.

\section{(J Neurogastroenterol Motil 2010;16:83-89)}

Key Words

Psuedo-obstruction, Chronic, Colon

\section{Introduction}

Chronic intestinal pseudo-obstruction (CIPO) is a rare, debilitating disorder characterized by obstructive symptoms mimicking mechanical obstruction without any lesion occluding the gut. ${ }^{1}$ Pseudo-obstruction could occur either with acute or chronic onset. Acute intestinal pseudo-obstruction, so called Ogilvie's syndrome, is the most common form. Acute intestinal pseudo-obstruction often occurs in critically ill patients or during the post- operative period. ${ }^{2,3}$ However, CIPO patients typically passes unrecognized for long periods of time before the definitive diagnosis is established, so affected individuals are often unable to maintain normal body weight and/or become malnourished. ${ }^{4}$ Furthermore, patients sometimes receive inadequate treatments or needless abdominal surgeries due to the delay in confirming the diagnosis. ${ }^{5}$ Therefore, it is very important for physicians to understand the pathophysiology, clinical features and treatment of this rare but debilitating disorder. We experienced a case of chronic pseudo-obstruction in the initial phase, which mimicked

Received: December 14th, 2009 Accepted: December 30th, 2009

(c) This is an Open Access article distributed under the terms of the Creative Commons Attribution Non-Commercial License (http://creativecommons. org/licenses/by-nc/3.0) which permits unrestricted non-commercial use, distribution, and reproduction in any medium, provided the original work is properly cited.

*Correspondence: Nayoung Kim, M.D., Ph.D.

Department of Internal Medicine, Seoul National University Bundang Hospital, 300 Gumi-dong, Bundang-gu, Seongnam, Gyeonggi-do 463-707, Korea

Financial support: None.

Tel: +82-31-787-7008, Fax: +82-31-787-4051, E-mail: nayoungkim49@empal.com

Conflicts of interest: None. 
an acute pseudo-obstruction, and treated through medical approach. He was compared to another recurrent and intractable case successfully treated with surgery and diagnosed as hypoganglionosis.

\section{Case Report}

\section{Case 1}

38-year-old male patient visited Emergency Room for ab- dominal pain and distension which were aggravated two days ago. Abdominal distension was developed 2 months ago but he did not visit any hospital since defecations with loose stools and gas passages were possible. He had no known history of any metabolic, neurological, cardiovascular or pulmonary diseases, previous abdominal or pelvic surgeries, and no history of abdominal cancer, inflammatory processes or trauma. No concomitant medication was taken. Physical examination revealed marked abdominal distension with hyperactive bowel sound, and he complained of mild tenderness on the entire abdomen without any rebound
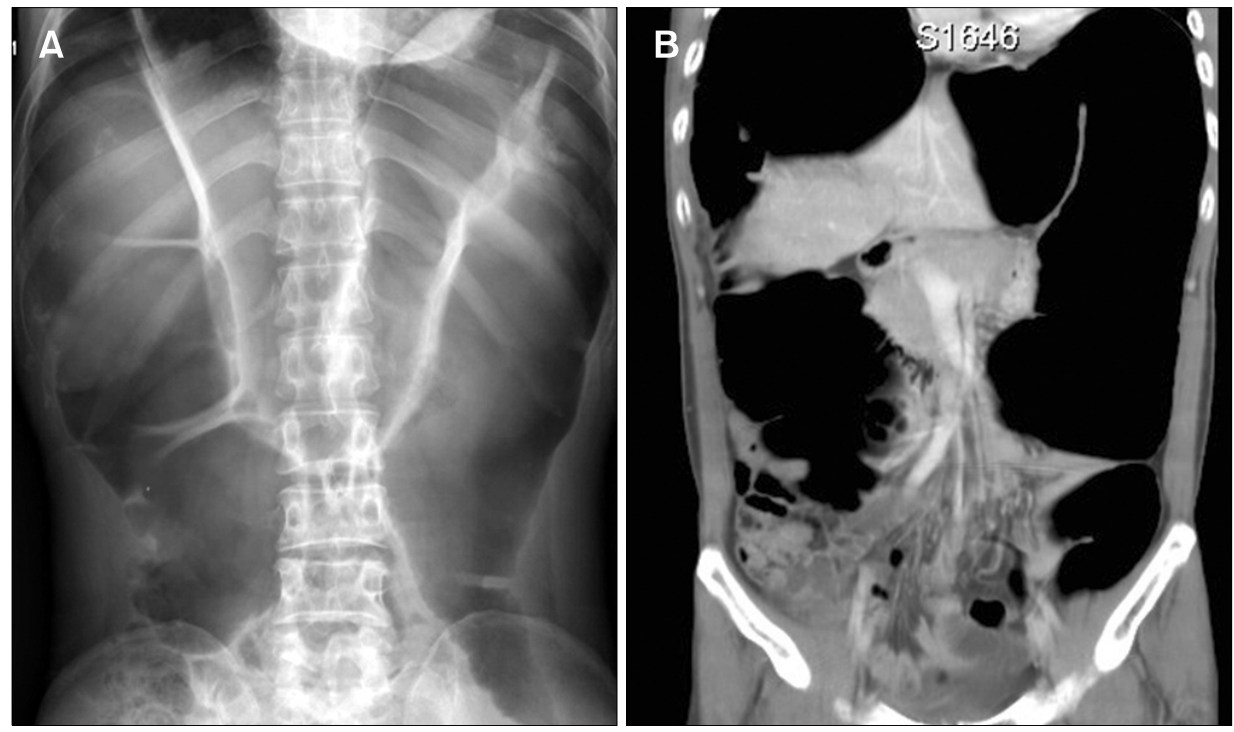

Figure 1. Radiologic findings of acute pseudo-obstruction case. Marked gaseous dilatation of the colon in simple abdomen (A) and gaseous distension of the colon from cecum to sigmoid colon in the $\mathrm{CT}$ axial scan, with well traced entire colon without a definitive obstructive lesion; narrowed segment is observed in the sigmoid colon (B).
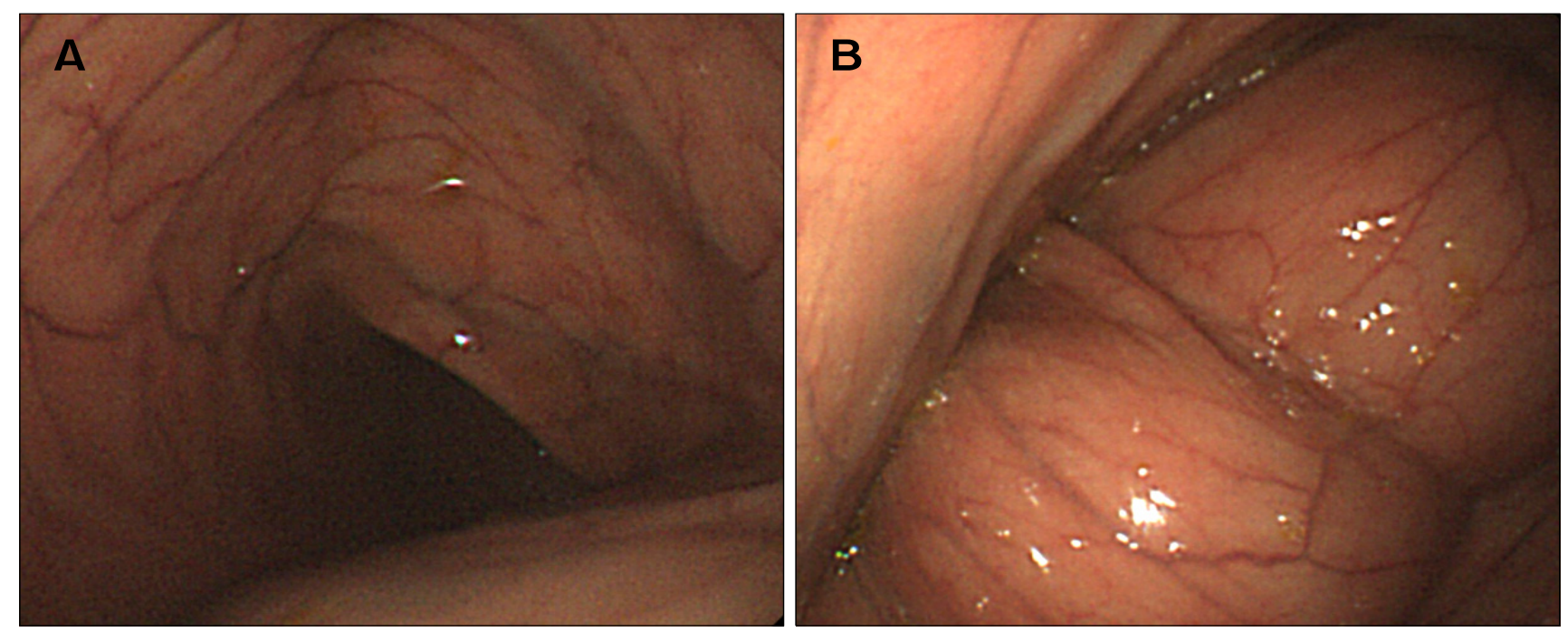

Figure 2. Colonoscopic finding in acute intestinal pseudo-obstruction. Marked dilation of colonic lumen without a mucosal or luminal lesion to cause mechanical obstruction (A); luminal kinking by gaseous distension is observed (B). 
tenderness. There was no sign of scar on the abdomen. Vital sign was stable and no fever was checked. The laboratory findings showed a hemoglobin of $14.7 \mathrm{~g} / \mathrm{dL}$, White blood cells $5,310 / \mathrm{mm}^{3}$ and platelets $265,000 / \mathrm{mm}^{3}$. The blood chemistry showed: a sodium of $142 \mathrm{mmol} / \mathrm{L}$, potassium $3.6 \mathrm{mmol} / \mathrm{L}$, chloride 105 $\mathrm{mmol} / \mathrm{L}$, calcium $8.6 \mathrm{mg} / \mathrm{dL}$ and albumin $4.4 \mathrm{~g} / \mathrm{dL}$. Fasting glucose level was $90 \mathrm{mg} / \mathrm{dL}$. Amylase and lipase were 51 and 59 $\mathrm{mg} / \mathrm{dL}$, respectively. Thyroid function test was normal; $\mathrm{T} 3$, free $\mathrm{T} 4$, and $\mathrm{TSH}$ were $0.7 \mathrm{ng} / \mathrm{mL}, 1.31 \mathrm{ng} / \mathrm{dL}$, and $2.86 \mu \mathrm{IU} / \mathrm{mL}$, respectively (all within normal range). There was no evidence of amyloidosis on colonoscopic biopsy. However, we did not perform tests for SLE or scleroderma because there were no clinical symptoms or physical signs of these diseases. Chest X-ray imaging showed normal findings. Marked gaseous dilatation above the pelvic cavity was shown in the simple abdominal X-ray image (Fig. 1A). Abdominal computed tomography revealed a marked distension from the ascending colon to the sigmoid colon whereas the entire colon was well traced without a definite obstructive
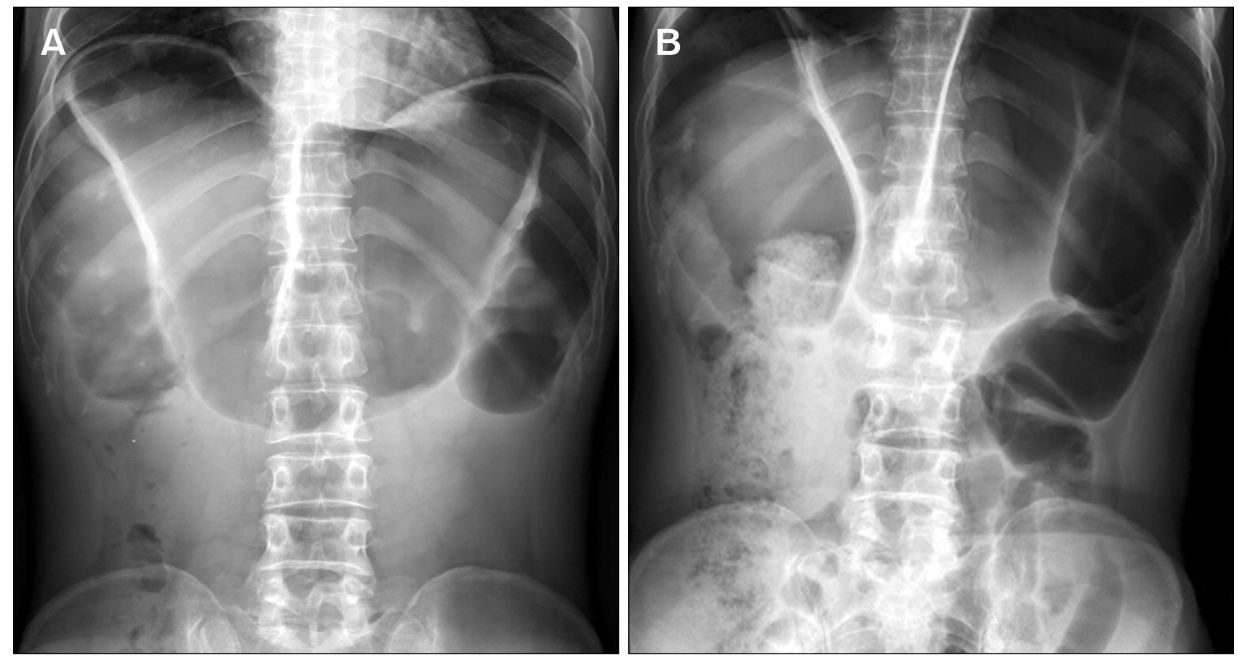

Figure 3. Follow-up radiologic finding after medical treatment. Gaseous distension is somewhat decreased, especially in the ascending and descending colon after i.v. neostigmine (A). Recent follow-up image shows decreased gaseous distension in the transverse colon after oral erythromycin, but ileus is still remained above the sigmoid colon (B).
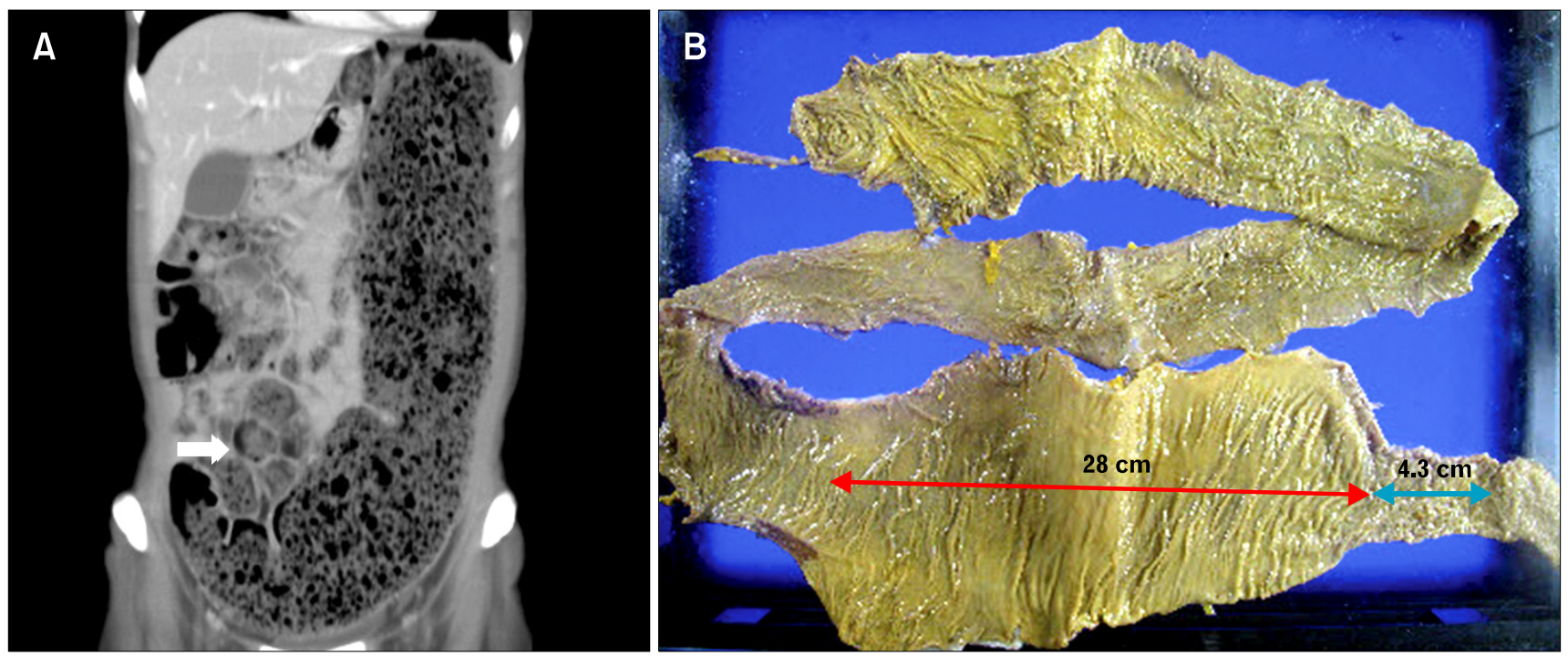

Figure 4. Radiologic and post-operative gross finding of chronic intestinal pseudo-obstruction case. Marked gaseous distension of the colon with profuse fecal materials from cecum to sigmoid colon was shown in the CT axial scan. However, the entire colon is well traced without a definitive obstructive lesion; narrowed segment is observed in the sigmoid colon (arrows) (A). Post operative gross findings shows 28 $\mathrm{cm}$ length of dilated segment (red arrow) and $4.3 \mathrm{~cm}$ length of narrowed segment (blue arrow) which is observed distal to the dilated segment (B). 
lesion. There was a narrowed segment in the sigmoid colon which was suggestive of transitional zone. No fluid collection or free air was shown in the abdominal cavity (Fig. 1B). Colonoscopy was performed for the evaluation of luminal lesions. Colonoscopy showed huge colonic dilatation (Fig. 2A) and kinking up to the cecum (Fig. 2B) but no obstructive, diverticular or mucosal lesions. Decompression with colonoscopic suction resulted in a decreased abdominal distension immediately. Randomized, multiple mucosal biopsies showed normal findings except mild ischemic changes due to the over-distension. Initially he was diagnosed as acute intestinal pseudo-obstruction, so called Ogilvie's syndrome, and intravenous neostigmine was tried with EKG monitoring. After intravenous administration of neostigmine (2.5 $\mathrm{mg}$ ) for 1 hour, side effects such as bradycardia, hypotension, atrial fibrillation developed and continuous infusion was stopped. Simultaneously, there was a dramatic evacuation of flatus and loose stools (Fig. 3A). However, abdominal distension developed again in the following day, and neostigmine (i.v. $2.0 \mathrm{mg}$ for 4 hours) was retried cautiously. Again there were evacuations of gases and loose stools without any side effects occurring abdominal distension developed in the next following day similar to the first trial of neostigmine. The symptom was endurable and ordinary activity was possible and the patient began to take $500 \mathrm{mg}$ of erythromycin three times per day. Although abdominal distension was sustained in spite of the maintenance of erythromycin (Fig. 3B), gas passages and defecations were possible every day and the degree of distension was rather mild. After two months he stopped taking erythromycin and started to take metoclopramide instead. He is doing well until now about five months from the discharge.

\section{Case 2}

43 year-old female visited Emergency Room for recurrent abdominal distension and pain. She suffered from constipation for 10 years which repeatedly aggravated and improved. From two months ago, it became worse and didn't improve. Physical examination in the emergency unit showed marked abdominal distension and mild abdominal tenderness. Bowel sound was mildly increased by the auscultation. There was no sign of scar on her abdominal wall. Vital signs were stable and no fever was detected. The laboratory findings showed a hemoglobin of 12.6 $\mathrm{g} / \mathrm{dL}$, platelets and white blood cells were $229 \times 10^{3} / \mu \mathrm{L}$ and $16.19 \times 10^{3} / \mu \mathrm{L}$, respectively. The blood chemistry showed: a sodium of $136 \mathrm{mmol} / \mathrm{L}$, potassium $4.1 \mathrm{mmol} / \mathrm{L}$, chloride 100 $\mathrm{mmol} / \mathrm{L}$, calcium $8.7 \mathrm{mg} / \mathrm{dL}$ and albumin $4.4 \mathrm{~g} / \mathrm{dL}$. Amylase was $21 \mathrm{mg} / \mathrm{dL}$ and other laboratory findings were within normal range. There was no history of any metabolic, neurological, cardiovascular or pulmonary diseases, previous abdominal or pelvic surgeries, and no history of abdominal cancer, inflammatory processes or trauma. No concomitant medication was taken. She never drank alcohol or smoked without any specific family history. After the admission, detailed medical tests were performed to find out the causes of abdominal pain and distention. There was no evidence of metabolic diseases such as diabetes, porphyria, or amyloidosis. Chest X-ray imaging showed normal findings. Marked gaseous distension in the colon, especially in the descending colon was seen in the simple abdominal X-ray image. Colon transit time test showed a delayed transit especially in the left colon. Anorectal manometric finding was normal. Abdominal computed tomography scan showed marked distension in the left colon and the sigmoid colon, but there was no specific lesion to induce mechanical obstruction or no fluid collection or free air in the peritoneal cavity (Fig. 4A). These radiologic and functional test suggested colonic pseudo-obstruction at the sigmoid colon level. Colonoscopy showed marked dilatation of the left and sigmoid colon, with non-specific ulcers in the transverse colon and rectum. Biopsies on ulcer lesions and random colonic areas showed non-specific changes. Under the impression of CIIPO surgical approach was chosen due to the recurrent symptoms and disturbed quality of life. Subtotal colectomy was performed. Dilated segment was $28 \mathrm{~cm}$ long and 4.3 $\mathrm{cm}$ length of narrowed segment was observed distal to the dilated segment (Fig. 4B). Markedly decreased number of mature myenteric ganglion cells, immature ganglion cells and decreased size of gangions in the myenteric plexus were observed in the narrowed segment (Fig. 5A). Immunoreactivities for c-Kit (Fig. 5B), synaptophysin (Fig. 5C), and Bcl-2 were markedly decreased in the myenteric plexus of the narrowed segment (Fig. $5 \mathrm{D})$, in which the pathologic findings confirmed hypoganglionosis as the cause of pseudo-obstruction. She is under a good nutritional status and symptoms have not recurred for 2 years after the surgery.

\section{Discussion}

Intestinal pseudo-obstruction is a disease characterized by failure of intestinal propulsion without a definite cause for mechanical obstruction. This disorder presents with a wide spectrum of clinical manifestations in respect to onset time of either acute or chronic and to the etiology as idiopathic or secondary. 

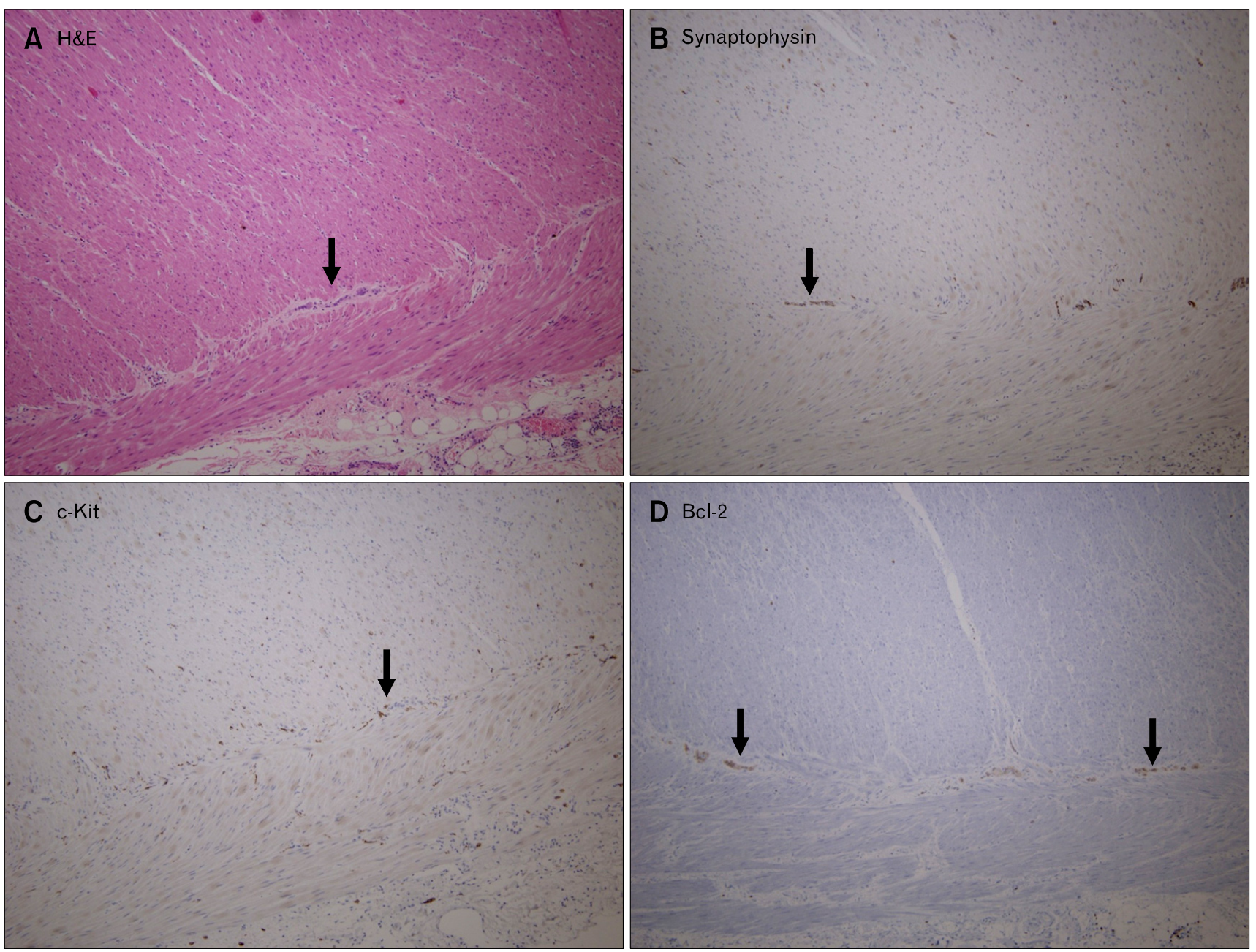

Figure 5. Pathologic finding of hypoganglionosis. Markedly decreased number of mature myenteric ganglion cells and decreased size (arrow) of ganglion are seen in the narrowed segment $(\mathrm{H} \& \mathrm{E} \times 100)(\mathrm{A})$. Decreased expression of synaptophysin $(\mathrm{B})$, c-Kit $(\mathrm{C})$, and Bcl-2 by immunohistochemistry in the myenteric plexus (arrow) $(\times 100)(\mathrm{D})$ is consistent with hypoganglionosis.

Acute intestinal pseudo-obstruction, also known as Ogilvie's syndrome, often occurs in hospitalized patients and is associated with a wide variety of medical and surgical conditions. ${ }^{2,3}$ Most of acute intestinal pseudo-obstruction occur in the colon and respond to conservative medical treatment, although perforation develops in a small number of patients with high mortality rate. ${ }^{6}$ Treatment modality such as medical therapy, colonoscopic decompression, or surgery should be individualized based on the severity of the patient's clinical status. ${ }^{3}$

In the first case, acute and first onset of pseudo-obstruction was developed and the patient was initially diagnosed as Ogilvie's syndrome. At first, the patient was evaluated for the possible causes of acute pseudo-obstruction and colonoscopic decompression was applied to the patient. No specific cause for acute pseudo-obstruction was found in spite of the comprehensive tests. Medical therapies such as neostigmine and erythromycin were only partially effective to the patient. Currently, the patient has been relatively doing well but mild sub-occlusive symptoms and signs were sustained during 5 months of follow-up period. As a result, the patient was considered to have undergone the first episode of CIPO. In the adult population, the first attack of pseudo-obstruction is often accompanied by a long history of non-specific, progressive symptoms like severe constipation. But an acute onset of the disease mimicking Ogilvie's syndrome also occurs in one-fourth of the cases as the first case patient. ${ }^{7}$

CIPO develops in either idiopathic or secondary form and the former comprises the majority of cases. In one study, systemic or metabolic causes of the disease were identified in only 4 of 77 
CIPO patients. ${ }^{7}$ Nevertheless, investigation of any secondary causes is necessary since correction of the secondary causes might be the only chance for cure of the CIPO. Many different diseases affect the control mechanisms of intestinal function, including intrinsic and extrinsic neural system as well as muscular system. ${ }^{4}$ The secondary causes that impair extrinsic nervous system can occur centrally or peripherally. The diseases affecting central nervous system are Parkinson syndrome, Shy-Drager syndrome, stroke, encephalitis, and neoplasm, whereas peripheral nervous system involves diabetes neuropathy, other neuropathies such as Hirschsprung, Chagas, and Von Reckling-hausen, as well as paraneoplastic syndromes, autoimmune diseases, or viral infections. ${ }^{4}$ Systemic muscular disorders like myotonic dystrophy and progressive systemic sclerosis could provoke damage to the intestinal smooth muscle cells and can also be the secondary causes of CIPO. ${ }^{4}$ Collagen vascular disease, Ehlers-Danlos syndrome, jejunal diverticulosis and radiation enteritis can be responsible for both neuronal and myogenic impairment. ${ }^{4}$ Furthermore, hypothyroidism, hypoparathyroidism, and celiac disease were reported to be the secondary causes of CIPO, even if the underlying mechanism remains undetermined. ${ }^{4}$

Chronic idiopathic intestinal pseudo-obstruction (CIIPO) is diagnosed when no underlying cause is found in spite of the detailed and thorough medical examination. Most cases of CIIPO in adults occur as sporadic forms, but some familial clusters were also reported as autosomal dominant, autosomal recessive or $\mathrm{X}$-linked transmission. ${ }^{8}$ In the present report, both patients were diagnosed as CIIPO without known causes for the secondary form of CIPO after the full examinations. Usually CIIPO is classified as neuropathic, mesenchymopathic and myopathic based on the pathogenesis affecting the enteric nervous system or smooth muscle cells, ${ }^{4,5}$ so the full-thickness biopsies of intestinal wall are mandatory to define the underlying mechanisms of CIIPO. Furthermore, neuropathic form of CIIPO is classified as degenerative or inflammatory based on the mechanism of damage to the enteric nervous system. Inflammatory neuropathies are diagnosed by infiltrations of dense inflammatory cells and damages to the myenteric plexus. Inflammatory cells in the myenteric plexus could be observed as lymphocyte predominant (lymphocytic myenteric ganglionitis) or eosinophil predominant (eosinophilic ganglionitis). ${ }^{9,10}$ Autoimmune mechanism is considered to involve in lymphocytic myenteric ganglionitis with several cases reported with positivity for anti-HuD anti-neuronal antibodies. ${ }^{11}$ Degenerative neuropathies are characterized by degeneration and loss of intrinsic neurons of the myenteric plexus. ${ }^{4}$
Typical pathologic findings reported in neurodegenerative CIIPO include various qualitative (neuronal swelling, intranuclear inclusions, axonal degeneration and others) and quantitative lesions (especially hypoganglionosis). ${ }^{4} \mathrm{Bcl}-2$ protein is widely expressed in the peripheral nervous system and is considered to play a major role in neuronal survival. In severe form of degenerative $\mathrm{CIIPO}, \mathrm{Bcl}-2$ protein is markedly reduced in the myenteric plexus as the second case patient. This pathologic finding suggests apoptosis in association with loss of ganglion cells in the myenteric plexus. ${ }^{9}$ In the second case patient, total colectomy was performed because of the recurrent, sub-occlusive events and ongoing severe constipation. The histological exam revealed marked decrease of ganglion cells in the myenteric plexus and decreased immunoreactivity for c-Kit, synaptophysin, and Bcl-2 confirming the diagnosis of hypoganglionosis.

In the first case, mild sub-occlusions were sustained in the radiologic exams with mild abdominal distension. We assume this patient could follow the clinical course of the second case which had shown the typical course of CIIPO. In the acute, sub-occlusive episodes of CIIPO, treatment should be the same as the mechanical obstruction with proper decompression, correction of fluid and electrolytes imbalance and appropriate nutritional support. Furthermore, erythromycin, somatostatin and neostigmine could aid the gastrointestinal motility, control visceral sensitivity and decrease the duration of sub-occlusive episodes. ${ }^{4,12,13}$ Erythromycin enhances upper gastrointestinal motility with a specific agonist action on the motilin receptors. ${ }^{12}$ Octerotide increases intestinal motility and decreases bacterial overgrowth. ${ }^{13}$ Combination of erythromycin and octreotide could be useful in controlling both the gastric emptying and the intestinal motility. ${ }^{14}$ Neostigmine is effective in Ogilvie's syndrome $^{15}$ and also in acute symptomatic relief of sub-occlusive episodes in CIIPO as in our case. Other medical options considered in the treatment of CIIPO are prokinetics, antibiotics, steroid and immunosuppressive agents. ${ }^{4}$

The effect of surgery is limited since CIIPO often involves the entire gastrointestinal tract with a progressive nature. ${ }^{16}$ As surgical approach can aggravate the underlying condition, it should be reserved for only carefully selected patients. However, surgical resection such as colectomy should be considered, especially in hypoganglionosis, ${ }^{17,18}$ as in the second case patient. In our hospital, five patients among 7 CIIPO were confirmed as hypoganglionosis during the last 6 years and all of them are doing well after the colectomy. Although the cases are few in numbers, these results suggest that hypoganglionosis is a frequent cause of 
CIIPO in Korea and colectomy offers a curable opportunity before bowel transplantation or death in the intractable cases, which is somewhat different from Western society.

In conclusion, we experienced two cases of CIIPO with different clinical features and treatment approaches. In the first case, the clinical feature was regarded as the first episode of CIPO mimicking Ogilvie's syndrome. Patient was successfully treated by medical approach in the acute stage with partial response. This patient could experience the recurrent and progressive nature of CIIPO. In the second case patient, typical clinical feature with recurrent episodes of CIIPO was shown, and she was successfully treated by total colectomy and was confirmed as hypoganglionosis, suggesting colectomy as a considerable and effective therapeutic option in the intractable and debilitating cases of hypoganglionosis.

\section{References}

1. Faulk DL, Anuras S, Christensen J. Chronic intestinal pseudoobstruction. Gastroenterology 1978;74:922-931.

2. Ogilvie H. Large-intestine colic due to sympathetic deprivation; a new clinical syndrome. Br Med J 1948;2:671-673.

3. Vanek VW, Al-Salti M. Acute pseudo-obstruction of the colon (Ogilvie's syndrome). An analysis of 400 cases. Dis Colon Rectum 1986;29:203-210.

4. Antonucci A, Fronzoni L, Cogliandro L, et al. Chronic intestinal pseudo-obstruction. World J Gastroenterol 2008;14:2953-2961.

5. Stanghellini V, Cogliandro RF, de Giorgio R, Barbara G, Salvioli B, Corinaldesi R. Chronic intestinal pseudo-obstruction: manifestations, natural history and management. Neurogastroenterol Motil 2007;19:440-452.

6. Rex DK. Colonoscopy and acute colonic pseudo-obstruction. Gastrointest Endosc Clin N Am 1997;7:499-508.
7. Stanghellini V, Cogliandro RF, De Giorgio R, et al. Natural history of chronic idiopathic intestinal pseudo-obstruction in adults: a single center study. Clin Gastroenterol Hepatol 2005;3:449-458.

8. De Giorgio R, Camilleri M. Human enteric neuropathies: morphology and molecular pathology. Neurogastroenterol Motil 2004;16: 515-531.

9. De Giorgio R, Sarnelli G, Corinaldesi R, Stanghellini V. Advances in our understanding of the pathology of chronic intestinal pseudo-obstruction. Gut 2004;53:1549-1552.

10. Schappi MG, Smith VV, Milla PJ, Lindley KJ. Eosinophilic myenteric ganglionitis is associated with functional intestinal obstruction. Gut 2003;52:752-755.

11. Caras S, Laurie S, Cronk W, Tompkins W, Brashear R, McCallum RW. Case report: pancreatic cancer presenting with paraneoplastic gastroparesis. Am J Med Sci 1996;312:34-36.

12. Emmanuel AV, Shand AG, Kamm MA. Erythromycin for the treatment of chronic intestinal pseudo-obstruction: description of six cases with a positive response. Aliment Pharmacol Ther 2004;19:687-694.

13. Soudah HC, Hasler WL, Owyang C. Effect of octreotide on intestinal motility and bacterial overgrowth in scleroderma. N Engl J Med 1991;325:1461-1467.

14. Verne GN, Eaker EY, Hardy E, Sninsky CA. Effect of octreotide and erythromycin on idiopathic and scleroderma-associated intestinal pseudoobstruction. Dig Dis Sci 1995;40:1892-1901.

15. Turegano-Fuentes F, Munoz-Jimenez F, Del Valle-Hernandez E, et al. Early resolution of Ogilvie's syndrome with intravenous neostigmine: a simple, effective treatment. Dis Colon Rectum 1997; 40:1353-1357.

16. Kim HY, Kim JH, Jung SE, Lee SC, Park KW, Kim WK. Surgical treatment and prognosis of chronic intestinal pseudo-obstruction in children. J Pediatr Surg 2005;40:1753-1759.

17. Georgescu EF, Vasile I, Georgescu AC. Intestinal pseudo-obstruction--a rare condition with heterogeneous etiology and unpredictable outcome. A case report. J Gastrointestin Liver Dis 2008;17:77-80.

18. Jung YJ, Kim YS, Lee KH, et al. A case of chronic idiopathic intestinal pseudoobstruction. Korean J Gastroenterol 1999;34:843848. 